\title{
Semi-Global Analysis of Relay Feedback Systems
}

\author{
Jorge M. Gonçalves ${ }^{1}$, Alexandre Megretski ${ }^{2}$, Munther A. Dahleh ${ }^{3}$ \\ Department of EECS, Room 35-401 \\ MIT, Cambridge, MA \\ jmg@mit.edu, ameg@mit.edu, dahleh@lids.mit.edu
}

\begin{abstract}
This paper presents semi-global sufficient stability conditions of limit cycles for relay feedback systems. Local stability conditions exist. These are based on analyzing the linear part of the Poincaré map. We know that when a certain limit cycle satisfies those local conditions, a neighborhood around the limit cycle exists such that any trajectory starting in this neighborhood converges to the limit cycle as time goes to infinity. However, tools to characterize this neighborhood do not exist. In this work, we present conditions, in the form of Linear Matrix Inequalities (LMIs), that guarantee the stability of a limit cycle in a reasonably large set around it. These results differ from previous local results as they take into account the high order terms of the Poincaré map.
\end{abstract}

\section{Introduction}

Analysis of linear systems in relay feedback is a classic field. The early work was motivated by relays in electromechanical systems and simple models of dry friction. Applications of relay feedback range from stationary control of industrial processes to control of mobile objects as used, for example, in space research. A vast collection of applications of relay feedback can be found in the first chapter of [9]. More recent examples include the delta-sigma modulator (as an alternative to conventional $\mathrm{A} / \mathrm{D}$ converters) and the automatic tuning of PID regulators. In the delta-sigma modulator, a relay produces a bit stream output whose pulse density depends on the applied input signal amplitude (see, for example, [1]). As for the automatic tuning of PID regulators, which are implemented in many industrial controllers, the idea is to determine some points on the Nyquist curve of a stable open loop plant by

\footnotetext{
${ }^{1}$ Research supported by the Portuguese "Junta Nacional de Investigação Científica e Tecnológica" under the program "PRAXIS XXI".

${ }^{2}$ Research supported in part by the NSF under grants ECS9410531, ECS-9796099, and ECS-9796033, and by the AFOSR under grant F49620-96-1-0123

${ }^{3}$ Research supported in part by the NSF under grant ECS9612558 and by the AFOSR under grant AFOSR F49620-950219 .
}

measuring the frequency of oscillation induced by a relay feedback (see, for example, [2]). One problem that needs to be solved here is the characterization of those systems that will give a unique global attractive limit cycle. This problem is important because it gives the class of systems when relay tuning can be used.

Although relay feedback is frequently used in practice, theoretical results exist only for very special cases. [4] and [9] are references that survey a number of analysis methods and results. An important property of relay feedback systems is that they often tend to a limit cycle. However, to tell in general if they actually converge to a limit oscillation is still an open problem. Rigorous results for analysis of local stability of relay feedback systems can be found, for example, in [3, 6]. In [3], necessary and sufficient conditions for local stability of limit cycles are presented. [6] emphasizes fast switches and their properties. For second-order systems, convergence analysis can be done in the phase-plane $[8,5]$. Stable second-order non-minimum phase processes can in this way be shown to have a globally attractive limit cycle. In [7] it is proved that this also holds for processes having an impulse response sufficiently close, in a certain sense, to a second-order non-minimum phase process.

Even though results like the ones in [3] provide particularly elegant mathematical solutions to many problems, they do have shortcomings. The linear difference equations used to describe the process are only approximations to the true behavior since all the nonlinearities were neglected. In many problems, like the sigma-delta modulator, it is important to verify at least semi-global stability and robustness of nonlinear oscillations. This can be done by taking explicitly into account the nonlinearities of the system. But, the methods available do not allow, in general, to verify these properties. In this paper, we will give semi-global sufficient stability conditions of limit cycles for relay feedback systems based on estimating those neglected nonlinearities. That is, we will present conditions that, when satisfied, guarantee the stability of a limit cycle in a reasonable large region around it. These conditions will be given in the form of linear matrix inequalities (LMIs) which can be efficiently solved using available computational tools. 
The remainder of this paper is organized as follows. Section 2 starts by reviewing some local stability results of limit cycles for relay feedback systems. In this same section, the problem we propose to solve is described in some detail. Section 3 presents the main result of this paper in one theorem followed by an example in section 4. The main result is proven in Section 5. Finally, conclusions and future work are discussed in Section 6.

\section{Previous work and problem description}

Consider a linear time-invariant system $P$ in feedback with a relay (see figure 1) given by

$$
\left\{\begin{array}{l}
\dot{x}=A x+B u \\
y=c x \\
u=-\operatorname{sgn}(y)
\end{array}\right.
$$

where $x \in \mathbb{R}^{n}$ and $u \in[-1,1]$ if $y=0$. Define the set $S$ as

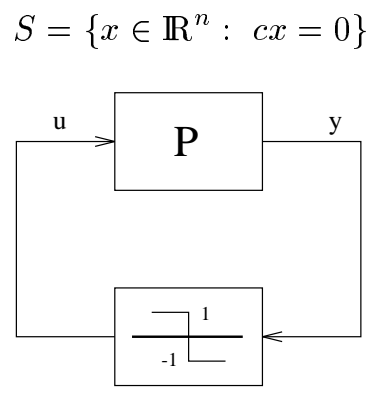

Figure 1: Relay Feedback System

In this paper, we assume $A$ is diagonalizable, i.e., there exists a set of $n$ linearly independent vectors $v_{i} \in \mathbf{C}^{n}$, each of which is an eigenvector of $A$. Without loss of generality, assume $A$ has the form $A=\operatorname{diag}\left(\lambda_{1} \cdots \lambda_{m} A_{1} \cdots A_{p}\right)$ where $\lambda_{i}, 1 \leq i \leq m$, are all the real eigenvalues of $A$, and matrices $A_{i}=$ $\left(\begin{array}{cc}\alpha_{i} & \beta_{i} \\ -\beta_{i} & \alpha_{i}\end{array}\right), 1 \leq i \leq p$, correspond to the complex eigenvalues $\alpha_{i} \pm j \beta_{i}$ of $A$. This form can always be obtained for a diagonalizable matrix by means of a similarity transformation. Let $I_{2}=\left(\begin{array}{ll}1 & 0 \\ 0 & 1\end{array}\right)$ and $Q^{T}$ denote the transpose of $Q$.

For simplification of results, and especially of proofs, throughout the this paper it is assumed that $A^{-1}$ exists. In practice, if this is not the case, we just need to make the appropriate modifications by noticing that $\left(e^{A t}-\right.$ I) $A^{-1}=\int_{0}^{t} e^{A \tau} d \tau$.

For a large class of processes, there will be limit cycle oscillations. The next theorem, proven in [3], gives necessary conditions for such limit cycles to occur.

Theorem 2.1 Consider the relay feedback system (1). Assume there exists a symmetric periodic solution $\gamma$ with period $2 t^{*}$. Then the following conditions hold

$$
g\left(t^{*}\right)=c\left(e^{A t^{*}}+I\right)^{-1}\left(e^{A t^{*}}-I\right) A^{-1} B=0
$$

and

$y(t)=c\left(e^{A t} x^{*}-\left(e^{A t}-I\right) A^{-1} B\right)>0$ for $0 \leq t<t^{*}$

Furthermore, the periodic solution $\gamma$ is obtained with the initial condition

$$
x(0)=x^{*}=\left(e^{A t^{*}}+I\right)^{-1}\left(e^{A t^{*}}-I\right) A^{-1} B
$$

In general, numerical procedures are needed to find the values of $t^{*}$ that satisfy (2). Once $t^{*}$ and the vector $x^{*}$ are found, they characterize a certain limit cycle $\gamma$ with period $2 t^{*}$ and a point $x^{*}$ that belongs to $\gamma$. We can now apply the following theorem from [3] which gives a necessary and sufficient condition for local stability of the limit cycle $\gamma$.

Theorem 2.2 Consider the relay feedback system (1). Assume there exists a symmetric periodic solution $\gamma$ with period $2 t^{*}$. Let $x^{*} \in S$ be the initial state that generates the periodic motion. The Jacobian of the Poincaré map is given by

$$
W=\left(I-\frac{v c}{c v}\right) e^{A t^{*}}
$$

where $v=A x^{*}-B$. The limit cycle $\gamma$ is locally stable if and only if $W$ has all its eigenvalues inside the unit disk.

This theorem gives local stability conditions for a given limit cycle. For instance, if $\gamma$ is proven to be a stable limit cycle then we know that there exist a neighborhood around $x^{*}$ such that any trajectory starting in this neighborhood will converge to the limit cycle $\gamma$. But, the theorem does not characterize in any way this neighborhood. This is because it only considers the Jacobian of the Poincaré map and neglects all its high order terms. Those high order terms carry the necessary information to characterize the stable neighborhood.

In this paper, the characterization of a stable region around $x^{*}$ of a locally stable limit cycle is considered. Semi-global sufficient conditions for the stability of limit cycles for relay feedback systems will be presented, i.e., conditions that, when satisfied, provide us with a reasonable large set of initial conditions such that any trajectory starting in that set converges to $\gamma$ as time goes to infinity.

\section{Main results}

Consider a locally stable limit cycle $\gamma$, with period $2 t^{*}$, obtained with the initial condition $x^{*} \in S$. This means 
that the trajectory starting at $x^{*}$ intersects for the first time the switching surface $S$ at $-x^{*}$ in $t^{*}$ time. Now, if $x^{*}$ is perturbed by $\Delta$ such that $x^{*}+\Delta \in S$ then the trajectory, starting at $x^{*}+\Delta$, will intersect $S$ at $-\left(x^{*}+\Delta_{1}\right)$ in $t^{*}+\delta$ time. Consider then the map $x^{*}+\Delta \rightarrow x^{*}+\Delta_{1}$ defined by $\Delta_{1}=T(\Delta)$ (this map is known as the Poincaré map). Throughout this paper, it will be assumed that this map is always continuous and smooth in the regions of interest. The map $T$ can be decomposed into linear and high order terms (see figure 2).

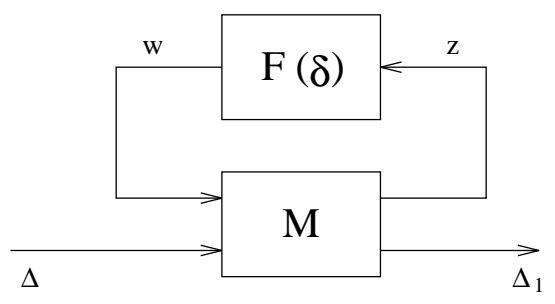

Figure 2: The $T$ map

Assume $|\delta| \leq r$ for some $r>0$ (that is, we start with the assumption that the time perturbation $\delta \in[-r, r]$ ). We have the following linear relation

$$
\left(\begin{array}{c}
\Delta_{1} \\
z
\end{array}\right)=M\left(\begin{array}{c}
\Delta \\
w
\end{array}\right)
$$

where $M$ is a $(3 n-1) \times(3 n-1)$ constant matrix (for a given $r>0$ ), and $w=F(\delta) z$ where $F(\delta)=$ $\operatorname{diag}\left(F_{1}(\delta), F_{2}(\delta)\right)$ represents the high order terms of $T$. We have the following quadratic inequality relating $w$ and $z$

$$
\left\|K^{-1} w\right\|_{D_{1}}^{2} \leq\|z\|_{D_{1}}^{2}
$$

where $K$ is a constant diagonal matrix (again, for a given $r>0$ ) and $D_{1}>0$ is a diagonal matrix. Consider the $n \times(n-1)$ matrix $\Pi$ (with $\left.\Pi^{T} \Pi=I\right)$ that maps one-to-one and onto vectors from $\mathbb{R}^{n-1}$ to $S$.

The following theorem is the main result of this paper. Under certain conditions (given in the form of LMIs) it provides a stable region $\bar{\Omega} \in S$ for $T$; that is, it characterizes a region for which any trajectory starting in it converges to the limit cycle $\gamma$ as time approaches infinity. This theorem provides then tools to analyze semi-global stability of relay feedback systems, adding more information to the previous local results in the sense that a reasonable large stable region around $\gamma$ can now be characterized.

Theorem 3.1 Consider the relay feedback system (1). Assume there exists a locally stable limit cycle $\gamma$, with period $2 t^{*}$ obtained with the initial condition $x^{*} \in S$. Pick $0 \leq \rho<1$. Given $r>0$, if

$$
\left[\begin{array}{cc}
\rho D & 0 \\
0 & K^{-2} D_{1}
\end{array}\right]-M^{T}\left[\begin{array}{cc}
D & 0 \\
0 & D_{1}
\end{array}\right] M>0
$$

is satisfied for some diagonal matrices $D, D_{1}>0$ with $D_{1}=\operatorname{diag}\left(D_{a}, D_{b}\right)$ and where both $D_{a}$ and $D_{b}$ have the form $\operatorname{diag}\left(d_{1}, \ldots, d_{m}, d_{1}^{c} I_{2}, \ldots, d_{p}^{c} I_{2}\right)$ then there exist a stable region $\bar{\Omega} \subset S$ for which any trajectory starting in this region will converge to the limit cycle $\gamma$, where $\bar{\Omega}=\left\{x \in \mathbb{R}^{n} \mid x=x^{*}+\Pi^{T} \Delta, \Delta \in \Omega\right\}$ with $\Omega=\{\Delta \in$ $\left.\mathbb{R}^{n-1} \mid\|\Delta\|_{D} \leq R\right\}$ and

$$
R=\min _{\delta \in[-r, r]}\left(\frac{\left|\bar{c} \frac{e^{A \delta}-I}{\delta} \bar{x}^{*}\right|}{\left\|\bar{c} e^{A \delta} \Pi\right\|_{D^{-1}}}\right) r
$$

A note on the initial choices of $\rho$ and $r$. The parameter $0 \leq \rho<1$ gives a measure on how fast the trajectory converges to $x^{*}$. A smaller $\rho$ implies a faster convergence. This means, we are more likely to find a larger stable region with a larger $\rho$ than with a smaller one. Thus, it is desirable to pick $\rho$ as close to 1 as possible, say $0.999 \cdots$. Now, for $r>0$ it is clear that a larger $r$ implies a larger $R$ and consequentially a larger region $\bar{\Omega} \subset S$. Therefore, we are interested in finding the largest $r>0$ such that the conditions of the theorem are still satisfied. We have then a one parameter search over $r>0$. Note also that due to the symmetry of $S$ around the origin, the region $\{-x \mid x \in \bar{\Omega}\}$ is also a stable region.

\section{Example}

Consider the following transfer function of a linear system

$$
P(s)=-\frac{(s+2.56)(s-1.56)}{(s+1)(s+2)(s+3)}
$$

which can be written in the state space form (1) with

$$
A=\left[\begin{array}{ccc}
-1 & 0 & 0 \\
0 & -2 & 0 \\
0 & 0 & -3
\end{array}\right], \quad B=\left[\begin{array}{c}
1 \\
-1 \\
1
\end{array}\right]
$$

and $c=\left[\begin{array}{lll}2 & 2 & -1\end{array}\right]$. Assume this system is in relay feedback. Solving (2) for $t^{*}>0$ we get $t^{*}=1.4$. This corresponds to $x^{*}=\left[\begin{array}{lll}0.60 & -0.44 & 0.32\end{array}\right]^{T} \in S$. Therefore, the closed loop system has a limit cycle obtained with the initial condition $x^{*} \in S$ and with period $2 t^{*}$. From theorem 2.2, we find that this limit cycle is locally stable. We are now ready to characterize a reasonable large region of stability around the limit cycle.

Solving the LMIs in theorem 3.1, we get

$$
D=\left[\begin{array}{cc}
5592 & 0 \\
0 & 2562
\end{array}\right]
$$

and $R=51.59$. Both $D$ and $R$ define a stable region $\Omega$ in $S$ that can be seen in figure 3 (the stable region is inside the ellipse centered at $\left.x^{*}\right)$. 


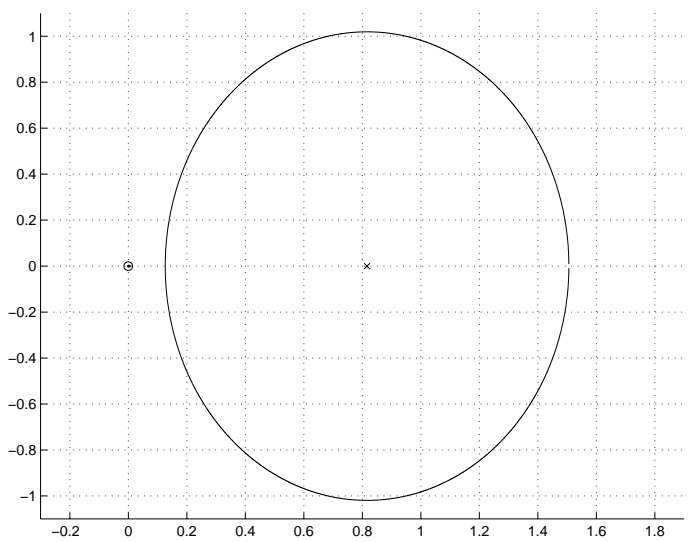

Figure 3: Stable region centered at $x^{*}$ denoted here by $\times$

\section{Proof of results}

The proof of theorem 3.1 is divided in three lemmas. The first lemma estimates the map $T$ (the Poincaré map). The second lemma proves that this map is stable in a region $\Omega$ characterized by $|\delta| \leq r$; that is, it is shown that $\Delta_{k}$ converges to zero as $k \rightarrow \infty$ where $T\left(\Delta_{k}\right)=\Delta_{k+1}$ for any point $\Delta_{0} \in \Omega$. Finally, the third lemma gives a complete characterization of this region $\Omega$ in terms of $r$.

We start by defining the Poincaré map for the relay feedback system (1). Let the map $T=T(x): S \rightarrow S$ be the map of a point $x$ belonging to the switching surface $S$ to the intersection of the trajectory, starting at $x$ and flowing with the system $P$, with the switching surface $S$ and reflecting the symmetry of $S$ about the origin.

Consider the relay feedback system (1). Assume there exists a locally stable limit cycle $\gamma$, with period $2 t^{*}$, obtained with initial condition $x^{*} \in S$. Note that $\gamma$ is a symmetric limit cycle about the origin. This means that if $x^{*} \in S$ is part of the limit cycle $\gamma$, then so it is $-x^{*}$. Let's now analyze the trajectory of the limit cycle starting at $x^{*} \in S$. Integrating the first equation of (1) with initial condition $x^{*}$ and $u\left(0^{+}\right)=-1$, we get

$$
x(t)=e^{A t}\left(x^{*}-A^{-1} B\right)+A^{-1} B
$$

Let $t^{*}>0$ be the first time the trajectory $x(t)$ intersects $S$, that is, the smallest $t^{*}>0$ that satisfies

$$
-x^{*}=x\left(t^{*}\right)=e^{A t^{*}}\left(x^{*}-A^{-1} B\right)+A^{-1} B
$$

Since $x\left(t^{*}\right) \in S$, we have $c x\left(t^{*}\right)=0$, or $c e^{A t^{*}}\left(x^{*}-\right.$ $\left.A^{-1} B\right)+c A^{-1} B=0$.

Consider the discrete-time system

$$
x_{k+1}=T\left(x_{k}\right)
$$

where $x_{0} \in S$ is a vector close enough to $x^{*}$. Since the limit cycle is locally stable, it implies that the sequence

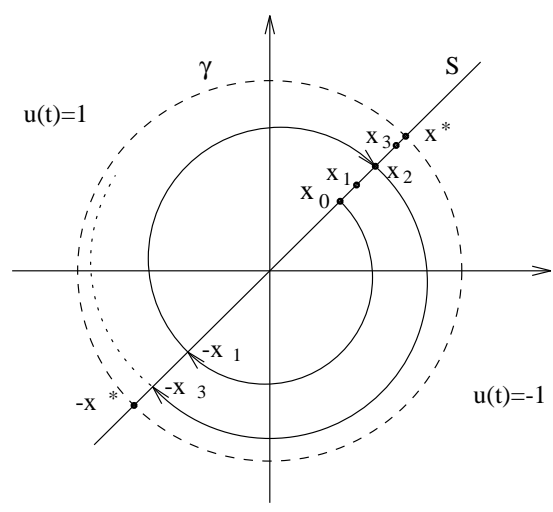

Figure 4: Convergence of a trajectory $x(t)$ to the limit cycle $\gamma$

$\left\{x_{k}\right\}$ converges to $x^{*}$ as $k \rightarrow \infty$ (see figure 4 ). Theorem 3.1 characterizes a region $\bar{\Omega} \subset S$ for which any $x_{0} \in \bar{\Omega}$ results in trajectories that converge to the limit cycle as time goes to infinity. The proof of the theorem is then based on showing that $T$ is stable in a certain region $\bar{\Omega} \subset S$.

Note that $x^{*}$ is an equilibrium point of (6). We will study this system when we perturb it by $\bar{\Delta} \in S$ from $x^{*}$. So, let $x_{0}=x^{*}+\Delta$. Applying $T$ to $x_{0}$ we get $x_{1}=$ $T\left(x_{0}\right)=x^{*}+\Delta_{1}$. In general, we have $x_{k}=T\left(x_{k-1}\right)=$ $x^{*}+\Delta_{k}$. Note that $\bar{\Delta}_{k} \in S$, and $S$ is a subspace of $\mathbb{R}^{n}$ of dimension $n-1$. Therefore, $\bar{\Delta}_{k}$ has only $n-1$ degrees of freedom. A diffeomorphism $\Pi: \mathbb{R}^{n-1} \rightarrow S \subset \mathbb{R}^{n}$ can then be constructed such that $\bar{\Delta}_{k}=\Pi \Delta_{k}$ where $\Delta_{k} \in \mathbb{R}^{n-1}$. Here $\Pi$ is a $n \times(n-1)$ matrix that can be chosen such that $\Pi^{T} \Pi=I$. In this case, $\Delta_{k}=$ $\Pi^{T} \bar{\Delta}_{k}$. Since $x^{*}$ is fixed, we will consider, without loss of generality, the discrete-time system $\Delta_{k+1}=T\left(\Delta_{k}\right)$. Note that 0 is now the equilibrium point of this system.

We are now ready for our first lemma where we estimate the map $T$.

Lemma 5.1 Assume that $|\delta| \leq r$ where $r>0$. The map $\Delta_{k+1}=T\left(\Delta_{k}\right)$ can be decomposed (see figure 2) in its linear part given by (3) where $M$ is a $(3 n-1) \times$ $(3 n-1)$ matrix that depends on $r$, plus its high order terms $w=F(\delta) z$ where $F(\delta)$ is a nonlinear function of $\delta$ and $r$. Moreover,

$$
\left\|K^{-1} w\right\|_{D_{1}}^{2} \leq\|z\|_{D_{1}}^{2}
$$

where $K$ is a constant diagonal matrix (for a given $r>$ $0)$, and $D_{1}=\operatorname{diag}\left(D_{a}, D_{b}\right)>0$ with both $D_{a}$ and $D_{b}$ having the form $\operatorname{diag}\left(d_{1}, \ldots, d_{m}, d_{1}^{c} I_{2}, \ldots, d_{p}^{c} I_{2}\right)>0$.

Proof: We have already seen what happens to the trajectory if we start at $x^{*}$. In this case, we have that $T(0)=0$. If we perturb $x^{*}$ by $\bar{\Delta}$ such that $x^{*}+\bar{\Delta} \in S$, then the point $x^{*}+\bar{\Delta}$ is mapped into $-\left(x^{*}+\bar{\Delta}_{1}\right) \in S$ 
in $t^{*}+\delta$ time. We have then the following equations

$$
\left\{\begin{array}{l}
-\left(x^{*}+\bar{\Delta}_{1}\right)=e^{A\left(t^{*}+\delta\right)}\left(x^{*}+\bar{\Delta}-A^{-1} B\right)+A^{-1} B \\
c e^{A\left(t^{*}+\delta\right)}\left(x^{*}+\bar{\Delta}-A^{-1} B\right)+c A^{-1} B=0
\end{array}\right.
$$

Using (5) and the fact that $\bar{\Delta}_{k}=\Pi \Delta_{k}$, we can write the last equations as

$$
\left\{\begin{array}{l}
\Delta_{1}=-\Pi^{T} e^{A t^{*}}\left(e^{A \delta}-I\right) \bar{x}^{*}-\Pi^{T} e^{A t^{*}} e^{A \delta} \Pi \Delta \\
\bar{c}\left(e^{A \delta}-I\right) \bar{x}^{*}+\bar{c} e^{A \delta} \Pi \Delta=0
\end{array}\right.
$$

where, for simplicity, we write $\bar{x}^{*}=x^{*}-A^{-1} B$ and $\bar{c}=c e^{A t^{*}}$.

The next step consists of computing the linear and high order terms of both equations. Let $F_{1 r}=\frac{e^{A r}+e^{-A r}}{2}$ and $F_{2 r}=\frac{e^{A r}-e^{-A r}}{2 r}$. The first equation of (7) can be rewritten as

$$
\Delta_{1}=\bar{L} \Delta+l \delta-\Pi^{T} e^{A t^{*}} F_{1}(\delta) \Pi \Delta-\Pi^{T} e^{A t^{*}} F_{2}(\delta) \bar{x}^{*} \delta
$$

where $\bar{L}=-\Pi^{T} e^{A t^{*}} F_{1 r} \Pi, l=-\Pi^{T} e^{A t^{*}} F_{2 r} \bar{x}^{*}, F_{1}(\delta)=$ $e^{A \delta}-F_{1 r}$, and $F_{2}(\delta)=\frac{e^{A \delta}-I}{\delta}-F_{2 r}$. The second equation can be written as

$$
\alpha \delta=-\beta \Delta-\bar{c} F_{2}(\delta) \bar{x}^{*} \delta-\bar{c} F_{1}(\delta) \Pi \Delta
$$

where $\alpha=\bar{c} F_{2 r} \bar{x}^{*}$ and $\beta=\bar{c} F_{1 r} \Pi$.

So, we have

$$
\left(\begin{array}{c}
\Delta_{1} \\
\alpha \delta
\end{array}\right)=L\left(\begin{array}{c}
\Delta \\
\delta
\end{array}\right)+\xi(\Delta, \delta)
$$

where $L=\left(\begin{array}{cc}\bar{L} & l \\ -\beta & 0\end{array}\right)$ and

$$
\xi(\Delta, \delta)=\left(\begin{array}{l}
K_{11} \\
K_{12}
\end{array}\right) F(\delta)\left(\begin{array}{c}
\Pi \Delta \\
\bar{x}^{*} \delta
\end{array}\right)
$$

with $K_{11}=-\Pi^{T} e^{A t^{*}}\left(\begin{array}{ll}1 & 1\end{array}\right), K_{12}=-\bar{c}\left(\begin{array}{ll}1 & 1\end{array}\right)$, and $F(\delta)=\operatorname{diag}\left(F_{1}(\delta), F_{2}(\delta)\right)$.

The next step will be to write the previous map in the form of the one in figure 2. Let $w=F(\delta) z$ with

$$
\begin{gathered}
z=\left(\begin{array}{c}
\Pi \Delta \\
\bar{x}^{*} \delta
\end{array}\right)=\left(\begin{array}{c}
z_{1} \\
z_{2}
\end{array}\right), \text { and }\left(\begin{array}{c}
\Delta_{1} \\
z_{1} \\
z_{2}
\end{array}\right)=M\left(\begin{array}{c}
\Delta \\
w
\end{array}\right) \text { where } \\
M=\left(\begin{array}{cc}
\bar{L}-l \frac{\beta}{\alpha} & l \frac{K_{12}}{\alpha}+K_{11} \\
\Pi & 0 \\
-\bar{x}^{*} \frac{\beta}{\alpha} & \bar{x}^{*} \frac{K_{12}}{\alpha}
\end{array}\right)
\end{gathered}
$$

We are now interested in estimating $\|w\|_{D_{2}}$, for some $D_{2}=D_{2}^{T}>0$, since $F(\delta)$ is a nonlinear function of $\delta$. For some $D_{1}=D_{1}^{T}>0$ and $D_{2}=D_{2}^{T}>0$ we have

$$
\begin{aligned}
\|w\|_{D_{2}}^{2} & =\left\|D_{2}^{1 / 2} F(\delta) D_{1}^{-1 / 2} D_{1}^{1 / 2} z\right\|_{2}^{2} \\
& \leq \sigma_{\max }^{2}\left(D_{2}^{1 / 2} F(\delta) D_{1}^{-1 / 2}\right)\|z\|_{D_{1}}^{2}
\end{aligned}
$$

If $D_{1}, D_{2}>0$ are picked such that they are diagonal with the structure $\operatorname{diag}\left(D_{a}, D_{b}\right)$ and with both $D_{a}$ and $D_{b}$ having the form $\operatorname{diag}\left(d_{1}, \ldots, d_{m}, d_{1}^{c} I_{2}, \ldots, d_{p}^{c} I_{2}\right)>0$ then $F F^{T}$ is a diagonal matrix that we write as $F F^{T}=$ $\operatorname{diag}\left(f_{1}, \ldots, f_{2 n}\right)$. Therefore, we have

$$
\begin{aligned}
\|w\|_{D_{2}}^{2} & \leq \sigma_{\max }\left(D_{2} F(\delta) F^{T}(\delta) D_{1}^{-1}\right)\|z\|_{D_{1}}^{2} \\
& \leq \max _{\delta \in[-r, r]} \sigma_{\max }\left(D_{2} F(\delta) F^{T}(\delta) D_{1}^{-1}\right)\|z\|_{D_{1}}^{2} \\
& =\sigma_{\max }\left(D_{2} K^{2} D_{1}^{-1}\right)\|z\|_{D_{1}}^{2}
\end{aligned}
$$

where $K^{2}=\operatorname{diag}\left(\max _{\delta} f_{1}(\delta), \ldots, \max _{\delta} f_{2 n}(\delta)\right)$. Since all the matrices in $\sigma_{\max }$ are diagonal, with $D_{1}=$ $\operatorname{diag}\left(d_{1}^{1}, \ldots, d_{2 n}^{1}\right), D_{2}=\operatorname{diag}\left(d_{1}^{2}, \ldots, d_{2 n}^{2}\right)$, and $K^{2}=$ $\operatorname{diag}\left(k_{1}^{2}, \ldots, k_{2 n}^{2}\right)$, the last inequality can be reduced to $\|w\|_{D_{2}}^{2} \leq p\|z\|_{D_{1}}^{2}$ where $p=\max _{i}\left\{\frac{d_{i}^{2}}{d_{i}^{1}} k_{i}^{2}\right\}$. In fact, $p=\frac{d_{i}^{2}}{d_{i}^{1}} k_{i}^{2}$ for all $i$ or otherwise there would exist at least one $i$ such that $\frac{d_{i}^{2}}{d_{i}^{1}} k_{i}^{2}<p$. Then one could decrease $d_{i}^{1}$ and obtain a smaller $\|z\|_{D_{1}}$. Hence, $\sum_{i} p \frac{d_{i}^{1}}{k_{i}^{2}} w_{i}^{2} \leq p\|z\|_{D_{1}}^{2}$ or $\left\|K^{-1} w\right\|_{D_{1}}^{2} \leq\|z\|_{D_{1}}^{2}$.

So, in lemma 5.1, the Poincaré map $T$ of a certain relay feedback system was decomposed in its linear part plus its high order terms around $x^{*}$. Now, we are interested in posing the following question: if we start at a certain point $x_{0} \in S$, with $\Delta_{0} \in \Omega$ (where, for now, $\Omega \subset \mathbb{R}^{n-1}$ is a region characterized by $|\delta| \leq r)$, will the sequence $\left\{x_{k}\right\}$ converge to $x^{*}$ as $k \rightarrow \infty$ ? Or, equivalent, will the sequence $\left\{\Delta_{k}\right\}$ converge to zero as $k \rightarrow \infty$ ? To answer this question we will use the following result:

Proposition 5.1 Let $T: \Omega \rightarrow \Omega$ be a function where $\Omega$ is a closed subset. If

$$
\|T(\Delta)\|_{D} \leq \rho\left\|_{\Delta}\right\|_{D}
$$

for all $\Delta \in \Omega$, some $0 \leq \rho<1$, and some $D=D^{T}>0$, then the sequence $\left\{\Delta_{k}\right\}$ defined as $\Delta_{k}=T\left(\Delta_{k-1}\right)$ and $\Delta_{0} \in \Omega$ will converge to zero as $k \rightarrow \infty$.

To see this, assume (8) is satisfied. Take any $\Delta_{0} \in \Omega$. Then $\left\|\Delta_{k}\right\|_{D}=\left\|T\left(\Delta_{k-1}\right)\right\|_{D} \leq \rho\left\|\Delta_{k-1}\right\|_{D} \leq \cdots \leq$ $\rho^{k}\left\|\Delta_{0}\right\|_{D}$. Now, as $k \rightarrow \infty$, the quantity $\rho^{k}$ approaches zero. This means that $\left\|\Delta_{k}\right\|_{D}$ can be made arbitrarily small by choosing $k$ sufficiently large.

In our case, the function is $T(\Delta)=\Delta_{1}$ (estimated in lemma 5.1) and the set $\Omega=\left\{\Delta \in \mathbb{R}^{n-1} \mid\|\Delta\|_{D}^{2} \leq R\right\}$ where $R>0$, unknown for now, depends on $r$. Note that we can define an equivalent subset of $S$ using the diffeomorphism $\Pi$, that is, $\bar{\Omega}=\left\{x \in \mathbb{R}^{n} \mid x=x^{*}+\right.$ $\left.\Pi^{T} \Delta, \Delta \in \Omega\right\} \subset S$.

The following lemma gives conditions in the form of LMIs that, when satisfied, guarantee the stability of $T$ in $\Omega$. 
Lemma 5.2 Let $0 \leq \rho<1$. The map $T$ is stable in $\Omega$ if there exists an $r>0$ such that

$$
\left[\begin{array}{cc}
\rho D & 0 \\
0 & K^{-2} D_{1}
\end{array}\right]-M^{T}\left[\begin{array}{cc}
D & 0 \\
0 & D_{1}
\end{array}\right] M>0
$$

for some diagonal matrices $D, D_{1}>0$.

Proof: To prove the stability of $T$ in $\Omega$ we need to show that

$$
\left\|\Delta_{1}\right\|_{D}^{2} \leq \rho\|\Delta\|_{D}^{2}
$$

for all $\Delta \in \Omega$ and some diagonal matrix $D>0$. Using the $S$-procedure, this is true if

$$
\rho\|\Delta\|_{D}^{2}-\left\|\Delta_{1}\right\|_{D}^{2} \geq\|z\|_{D_{1}}^{2}-\left\|K^{-1} w\right\|_{D_{1}}^{2}
$$

is satisfied for all $\Delta \in \mathbb{R}^{n-1}, w \in \mathbb{R}^{n}$ and some diagonal positive definite matrices $D \in \mathbb{R}^{n-1 \times n-1}$, $D_{1} \in \mathbb{R}^{2 n \times 2 n}$. The desired result can be obtained by using (3) and simple manipulation of the last inequality.

We are left with the characterization of $\Omega$. Note that the assumption of the time perturbation $\delta$ being bounded by $r>0$ defines automatically a region $\bar{\Omega} \subset S$ for which the trajectory starting at any point in this region takes less than $t^{*}+r$ time and more than $t^{*}-r$ time to intersect for the first time the switching surface $S$. The next lemma gives an estimative on the size of this region.

Lemma 5.3 For any $D=D^{T}>0$ and $r>0$, there exists an $R>0$ such that if $\|\Delta\|_{D} \leq R$ then $|\delta| \leq r$. Such $R>0$ is given by

$$
R=\min _{\delta \in[-r, r]}\left(\frac{\left|\bar{c} \frac{e^{A \delta}-I}{\delta} \bar{x}^{*}\right|}{\left\|\bar{c} e^{A \delta} \Pi\right\|_{D^{-1}}}\right) r
$$

Proof: Let $r>0$. From the second equation of (7) we have $\left|\bar{c} \frac{e^{A \delta}-I}{\delta} \bar{x}^{*}\right||\delta|=\left|\bar{c} e^{A \delta} \Pi \Delta\right|$ or

$$
|\delta| \leq \frac{\left\|\bar{c} e^{A \delta} \Pi\right\|_{D^{-1}}}{\left|\bar{c} \frac{e^{A \delta}-I}{\delta} \bar{x}^{*}\right|}\|\Delta\|_{D}=\frac{1}{f(\delta)}\|\Delta\|_{D}
$$

for all $\Delta$ and $\delta=\tilde{\delta}(\Delta)$. It is easy to see that if $\Delta=0$ then $\delta=0$. Now, due to the fact that $f(\delta)$ is continuous on $[0, r]$ (by initial assumption in the paper), one can find an $\hat{R}>0$ small enough such that

$$
\|\Delta\|_{D} \leq \hat{R} \Rightarrow|\delta| \leq r
$$

because a small increase in $\|\Delta\|_{D}$ must correspond to a small increase in $|\delta|$. Let $\hat{R}=\min _{\delta \in[-r, r]} f(\delta) \hat{r}$ where $0<\hat{r} \leq r$ is small enough. Then $\|\Delta\|_{D} \leq \hat{R} \Rightarrow|\delta| \leq \hat{r}$.

Now, if we increase $\hat{r}$ to $r$, we have $|\delta| \leq r$ which means that $(9)$ is not violated. Therefore we found $R=\min _{\delta \in[-r, r]} f(\delta) r$ for which $\|\Delta\|_{D} \leq R \Rightarrow|\delta| \leq r$.

\section{Conclusions and future work}

This paper presented conditions for semi-global stability of limit cycles for relay feedback systems. These conditions were given in the form of linear matrix inequalities, which can be solved using available and efficient computational tools. The idea behind the development of these conditions consisted of three steps:

1. separation of the Poincaré map in its linear part plus its high order terms (first part of lemma 5.1);

2. estimation of those high order terms (second part of lemma 5.1);

3. characterization of a reasonable large region in the state space for which any trajectory starting in this region converges to the locally stable limit cycle (lemmas 5.2 and 5.3).

There are still many open problems following this work. One is the extension of the results from this paper to other types of nonlinearities (like saturations, etc.). To be more general, these results should be extended to cover stability analysis of limit cycles for uncertain systems in relay feedback, piecewise linear systems, and certain classes of piecewise nonlinear systems.

\section{References}

[1] S. H Ardalan and J. J. Paulos. An analysis of nonlinear behavior in delta-sigma modulators. IEEE Trans. Circuits and Sys., 6:33-43, 1987.

[2] K. J. Ȧström and T. Hagglund. Automatic tuning of simple regulators with specifications on phase and amplitude margins. Automatica, 20:645-651, 1984. [3] Karl J. Áström. Oscillations in systems with relay feedback. The IMA Volumes in Mathematics and its Applications: Adaptive Control, Filtering, and Signal Processing, 74:1-25, 1995.

[4] D. P. Atherton. Nonlinear Control Engineering. Van Nostrand, 1975.

[5] John Guckenheimer and Philip Holmes. Nonlinear Oscillations, Dynamical Systems, and Bifurcations of Vector Fields. Springer-Verlag, N.Y., 1983.

[6] Karl H. Johansson, Anders Rantzer, and Karl J . Aström. Fast switches in relay feedback systems. To Appear in Automatica, 1998.

[7] A. Megretski. Global stability of oscillations induced by a relay feedback. In Preprints 9th IFAC World Congress, Budapest, Hungary, E:49-54, 1996.

[8] Yasundo Takahashi, Michael J. Rabins, and David M. Auslander. Control and Dynamic Systems. Addison-Wesley, Reading, Massachusetts, 1970.

[9] Ya. Z. Tsypkin. Relay control systems. Cambridge University Press, Cambridge, UK, 1984. 